\title{
In vitro potency of amikacin and comparators against E. coli, K. pneumoniae and $P$. aeruginosa respiratory and blood isolates
}

\author{
Christina A. Sutherland ${ }^{1}$, Jamie E. Verastegui ${ }^{1}$ and David P. Nicolau ${ }^{1,2^{*}}$
}

\begin{abstract}
Background: The purpose of this study was to define the potency of amikacin and comparator agents against a collection of blood and respiratory nosocomial isolates implicated in ICU based pulmonary infections gathered from US hospitals.

Methods: Minimum inhibitory concentrations of amikacin, aztreonam, cefepime, ceftazidime, ceftolozane/tazobactam, ceftriaxone, ciprofloxacin, imipenem, meropenem, piperacillin/tazobactam and tobramycin were tested against 2460 Gram-negative isolates. Amikacin had $96 \%$ susceptibility against the combined E. coli and K. pneumoniae isolates and $95 \%$ susceptibility against P. aeruginosa.

Results: Ninety-six percent of all of isolates tested were susceptible (i.e., MICs $\leq 16 \mathrm{mg} / \mathrm{L}$ ) to amikacin by current laboratory standards which demonstrates a high level of activity to combat infections caused by these organisms including ESBL, MDR, $\beta$-lactam and fluoroquinolone resistant strains. Moreover, $99 \%$ of all organisms had amikacin MICs $\leq 64 \mathrm{mg} / \mathrm{L}$.

Conclusions: Overall, these data highlight the continued potency of amikacin and suggest that the achievable lung concentrations of approximately $5000 \mathrm{mg} / \mathrm{L}$ with the administration of the amikacin by inhalation (Amikacin Inhale, BAY41-6551) will exceed the MICs typically observed for P. aeruginosa, E. coli and K. pneumoniae in the hospital setting.
\end{abstract}

Keywords: Amikacin, E. coli, K. pneumoniae, P. aeruginosa

\section{Background}

The management of hospital-acquired pneumonia (HAP) and ventilator-associated pneumonia (VAP) has been made increasingly difficult due to the emergence of resistance and the potential for reduced antibiotic lung penetration in the intubated patient. HAP continues to be the second most common cause of nosocomial infections in the United States and is associated with increases in hospital length of stay, healthcare costs and represents a major cause of mortality especially in critically ill patients $[1,2]$. VAP, a subset of HAP, that occurs in mechanically ventilated patients more than $48 \mathrm{~h}$ after tracheal intubation and occurs in $9-40 \%$ of mechanically

\footnotetext{
*Correspondence: david.nicolau@hhchealth.org

${ }^{1}$ Center for Anti-Infective Research and Development, Hartford Hospital, 80 Seymour Street, Hartford, CT 06102, USA

Full list of author information is available at the end of the article
}

ventilated patient's making it among the most frequent infections in the ICU $[1,3]$.

Gram-negative bacteria are responsible for a substantial proportion of HAP and VAP infections. Pseudomonas aeruginosa, along with the Enterobacteriacae, Escherichia coli and Klebsiella pneumoniae are amongst the most common etiological organisms representing approximately two-thirds of causative agents $[4,5]$. Nosocomial pneumonia caused by $P$. aeruginosa, E. coli and $K$. pneumoniae continues to pose significant challenges in US hospitals due to their prevalence and the acquisition of numerous antimicrobial resistance mechanisms. As a result, the selection of empirical antibiotic therapy in patients with nosocomial respiratory tract infections has become increasingly challenging as the number of potentially effective agents has been reduced due to evolving resistance. Yet more challenging is delivering sufficiently 
high antibiotic concentrations to the lung as many parenteral therapies have poor or variable penetration. The delivery of antibiotics directly to the site of infection presents a unique clinical opportunity to enhance patient outcomes by achieving high local concentrations that overcome resistance while minimizing the potential for toxicity associated with systemic administration.

Amikacin Inhale (BAY41-6551) is a reformulated solution of amikacin (AMK) combined with a drug-delivery module that is currently under phase III study as an adjunctive therapy for the treatment of Gram-negative pneumonia in intubated and mechanical ventilated patients. In vitro pharmacodynamic models evaluating the achievable epithelial lining fluid (ELF) concentrations after the administration of AMK inhalation against Gram-negative organisms demonstrated rapid and sustainable bactericidal killing of AMK both alone and in combination with systemic exposures of meropenem when AMK MICs were $\leq 256 \mathrm{mg} / \mathrm{L}$ [6]. Our objective was to define the potency and MIC distribution of AMK against a US collection of $P$. aeruginosa, E. coli, $K$. pneumoniae nosocomial isolates and relate these data to achievable lung concentrations of the compound when delivered via the aerosol route.

\section{Methods}

Fifty US hospitals, 41 teaching and 9 community provided non-duplicate nosocomial blood and respiratory isolates of E. coli, K. pneumoniae and P. aeruginosa from adult inpatients. Additionally, five of these hospitals also provided S. maltophilia respiratory isolates. Organisms were identified at each participating site using the standard methods. The isolates were transferred to trypticase soy agar slants for shipping to the Center for Anti-Infective Research and Development, Hartford Hospital, Hartford, CT, USA. Collection occurred from 2013 into 2014.

Clinical Laboratory Standards Institute (CLSI) defined broth microdilution methods were employed to determine minimum inhibitory concentration (MICs) for AMK, aztreonam (ATM), cefepime (FEP), ceftazidime (CAZ), ceftolozane/tazobactam (C/T), ceftriaxone (CRO), ciprofloxacin (CIP), imipenem (IPM), meropenem (MEM), piperacillin/tazobactam (TZP) and tobramycin (TOB) [7]. Antibiotics were purchased from Sigma (St. Louis, MO) except for $\mathrm{C} / \mathrm{T}$ which was provided by Cubist Pharmaceuticals. Quality control was performed on each batch of MIC testing using $E$. coli 25922 and P. aeruginosa 27853 as defined by CLSI. All transfer and colony counts were performed on trypticase soy agar plates containing $5 \%$ blood. CLSI and FDA breakpoints were used to define susceptibility. For $\mathrm{C} / \mathrm{T}$ the FDA breakpoints of $2 \mathrm{mg} / \mathrm{L}$ for Enterobacteriaceae and $4 \mathrm{mg} / \mathrm{L}$ for $P$. aeruginosa were utilized [8]. Isolates that were non-susceptible to AMK (i.e., $\geq 32 \mathrm{mg} / \mathrm{L}$ ) by current laboratory definitions were repeated and confirmed.

Pseudomonas aeruginosa were identified as multidrug resistant (MDR) if they displayed resistance to 3 or more classes as represented by the following phenotypic resistance profiles: CIP (MIC $\geq 4 \mathrm{mg} / \mathrm{L})$, IPM (MIC $\geq 8 \mathrm{mg} / \mathrm{L}$ ), $\mathrm{CAZ}(\mathrm{MIC} \geq 32 \mathrm{mg} / \mathrm{L}), \mathrm{TZP}(\mathrm{MIC} \geq 128 \mathrm{mg} / \mathrm{L})$ and TOB $(\mathrm{MIC} \geq 16 \mathrm{mg} / \mathrm{L})[9]$.

Escherichia coli and K. pneumoniae were tested for extended spectrum $\beta$-lactamases (ESBL) production if they had an MIC of $\geq 1$ to $2 \mathrm{mg} / \mathrm{L}$ of the following: ATM, CRO or CAZ. CLSI defined ESBL confirmation studies were then undertaken using additional MIC testing with CAZ, CAZ with clavulanate, cefotaxime and cefotaxime with clavulanate [7].

Isolates testing non-susceptible to ertapenem, imipenem, or meropenem were evaluated for carbapenemase production using the CarbaNP test [10].

\section{Results}

Hospitals provided nosocomial blood $(\mathrm{n}=1118)$ and respiratory isolates $(\mathrm{n}=527)$ of E. coli $(\mathrm{n}=811)$ and $K$. pneumoniae $(\mathrm{n}=835)$. Demographic from these patients are shown in Table 1. Rank order \% susceptibility for the Enterobacteriaceae, was as follows: AMK $96 \%$, IPM $96 \%$, MEM $96 \%$, C/T $94 \%$, TZP 88 \%, FEP $87 \%$, CAZ $85 \%$, ATM $85 \%$, TOB $84 \%$, CRO $84 \%$ and CIP $74 \%$ (Table 2). For AMK $96 \%$ of these organisms had MICs $\leq 16 \mathrm{mg} / \mathrm{L}$, $2 \% 32 \mathrm{mg} / \mathrm{L}$ and $2 \% \geq 64 \mathrm{mg} / \mathrm{L}$ (Fig. 1). Comparing AMK against both FEP $(\mathrm{n}=216)$ and TZP resistant $(\mathrm{n}=191)$ isolates only $2 \%$ of the organisms had MICs $\geq 128 \mathrm{mg} / \mathrm{L}$, respectively. Interesting one $E$. coli isolate was found to have an AMK MIC of $65,536 \mathrm{mg} / \mathrm{L}$. Isolates from blood had a slightly higher (1-7 \%) susceptibility for all agents tested than the respiratory isolates.

Of the $1646 \mathrm{E}$. coli and K. pneumoniae collected, 173 isolates were confirmed to be ESBL positive. Of these ESBL isolates, $87 \%$ were found to have MICs $\leq 16 \mathrm{mg} / \mathrm{L}$ to AMK. When considering only the Enterobacteriaceae confirmed ESBL positive isolates the rank order susceptibility of the conventional agents was as follows: MEM $95 \%$, IPM 94 \%, AMK $87 \%$, C/T $79 \%$, TZP $62 \%$, TOB $39 \%$, FEP $16 \%$, CIP $16 \%$, CAZ $14 \%$, ATM $9 \%$ and CRO $5 \%$. For AMK $87 \%$ of these organisms had MICs $\leq 16 \mathrm{mg} / \mathrm{L}, 9 \% 32 \mathrm{mg} / \mathrm{L}$ and $2 \% \geq 64 \mathrm{mg} / \mathrm{L}$. Of the total Enterobacteriaceae isolates collected three E. coli and $37 \mathrm{~K}$. pneumoniae were carbapenemase-producing as defined by the CarbaNP test. The susceptibility of AMK, CIP and TOB for these isolates were 53, 15 and $10 \%$, respectively. All of the other agents had $<9 \%$ 
Table 1 Age, hospital location and infection site of patients with $E$. coli, K. pneumoniae and $P$. aeruginosa isolates

\begin{tabular}{llllll}
\hline $\begin{array}{l}\text { Age range } \\
\text { (years) }\end{array}$ & No. of & \multicolumn{5}{l}{ Percentage of isolates from: } \\
\cline { 3 - 6 } & patients & $\begin{array}{l}\text { ICU } \\
\text { (\%) }\end{array}$ & $\begin{array}{l}\text { Non-ICU } \\
(\%)\end{array}$ & $\begin{array}{l}\text { Respiratory } \\
(\%)\end{array}$ & $\begin{array}{l}\text { Blood } \\
(\%)\end{array}$ \\
\hline $18-30$ & 210 & 37 & 63 & 63 & 37 \\
$31-40$ & 166 & 39 & 61 & 59 & 41 \\
$41-50$ & 244 & 44 & 56 & 50 & 50 \\
$51-60$ & 470 & 42 & 58 & 50 & 50 \\
$61-70$ & 597 & 65 & 35 & 51 & 49 \\
$>70$ & 773 & 34 & 66 & 43 & 57 \\
Total & 2460 & 45 & 55 & 50 & 50 \\
\hline
\end{tabular}

susceptibility. For AMK $53 \%$ of these organisms had MICs $\leq 16 \mathrm{mg} / \mathrm{L}, 15 \% 32 \mathrm{mg} / \mathrm{L}$ and $29 \% 64 \mathrm{mg} / \mathrm{L}$ and $3 \% 256 \mathrm{mg} / \mathrm{L}$.

Hospitals provided 814 nosocomial blood and respiratory isolates of $P$. aeruginosa. Rank order \% susceptibility was as follows: AMK $95 \%$, C/T $95 \%$, TOB $90 \%$, CAZ $74 \%$, FEP 73 \%, MEM $71 \%$, CIP $68 \%$, ATM $67 \%$, TZP $67 \%$ and IPM $62 \%$ (Table 3). For AMK $95 \%$ of these organisms had MICs $\leq 16 \mathrm{mg} / \mathrm{L}, 2 \% 32 \mathrm{mg} / \mathrm{L}, 2 \%$ $64 \mathrm{mg} / \mathrm{L}$ and $1 \% \geq 128 \mathrm{mg} / \mathrm{L}$ (Fig. 1). Comparing AMK against MEM resistant isolates $(\mathrm{n}=236), 91 \%$ of these organisms had MICs $\leq 16 \mathrm{mg} / \mathrm{L}, 6 \% 32-64 \mathrm{mg} / \mathrm{L}$ and $3 \% \geq 128 \mathrm{mg} / \mathrm{L}$. Comparing AMK against FEP resistant isolates $(\mathrm{n}=222), 89 \%$ of these organisms had MICs $\leq 16 \mathrm{mg} / \mathrm{L}, 9 \% 32-64 \mathrm{mg} / \mathrm{L}$ and $2 \% \geq 128 \mathrm{mg} / \mathrm{L}$. Comparing AMK against TZP resistant isolates $(\mathrm{n}=267)$, $91 \%$ of these organisms had MICs $\leq 16 \mathrm{mg} / \mathrm{L}, 7 \%$ $32-64 \mathrm{mg} / \mathrm{L}$ and $1 \% \geq 128 \mathrm{mg} / \mathrm{L}$. Like the Enterobacteriaceae, $P$. aeruginosa isolates from blood had a higher (3-14) \% susceptibility for all agents tested than the respiratory isolates. The susceptibility profile of ATM, FEP, CAZ, IPM, MEM and TZP was 10 to $14 \%$ higher from patients with blood cultures.

Fourteen percent $(\mathrm{n}=116)$ of the $P$. aeruginosa population was defined as MDR. In this subset of MDR isolates, rank order $\%$ S was as follows: AMK $87 \%$, C/T $78 \%$, TOB $52 \%$, ATM $19 \%$, MEM $17 \%$, CAZ $16 \%$, FEP $13 \%$, IPM $11 \%$, CIP $11 \%$ and TZP $6 \%$. While the $\mathrm{MIC}_{90}$ was 32 for AMK, $23 \%$ of these organisms had MICs $\leq 4 \mathrm{mg} / \mathrm{L}, 38 \%$ $8 \mathrm{mg} / \mathrm{L}, 26 \% 16 \mathrm{mg} / \mathrm{L} 5 \% 32 \mathrm{mg} / \mathrm{L}, 4 \% 64 \mathrm{mg} / \mathrm{L}, 3 \%$ $256 \mathrm{mg} / \mathrm{L}$ and $1 \% \geq 512 \mathrm{mg} / \mathrm{L}$. Of note $2 P$. aeruginosa isolates were found to have an AMK MIC of $65,536 \mathrm{mg} / \mathrm{L}$.

The AMK distribution for S. maltophilia $(\mathrm{n}=45)$ was $29 \%$ of these organisms had MICs $\leq 16 \mathrm{mg} / \mathrm{L}, 16 \%$ $32 \mathrm{mg} / \mathrm{L}, 9 \%$ 64, $24 \% 128 \mathrm{mg} / \mathrm{L}, 15 \% 256 \mathrm{mg} / \mathrm{L}$ and $7 \%$ $\geq 512 \mathrm{mg} / \mathrm{L}$.
To determine if the susceptibility of these organisms are influenced by the source of infection and location of the patient, we evaluated the \% susceptibility of ICU versus Non-ICU patients. The Enterobacteriaceae had no more than a $2 \%$ difference in \% susceptibility between the ICU $(\mathrm{n}=539)$ and Non-ICU $(\mathrm{n}=1106)$ patients. For the $P$. aeruginosa, we noted differing susceptibility profiles derived from the ICU and non-ICU setting. The susceptibility profile of ATM, FEP, CAZ, IPM, MEM and TZP was $5-11 \%$ lower from patients in the ICU. In contrast, $\leq 3 \%$ difference in was observed between the potency of ICU and non-ICU P. aeruginosa for AMK, $\mathrm{C} / \mathrm{T}, \mathrm{CIP}$ and TOB.

\section{Discussion}

Hospital-acquired pneumonia (HAP) and ventilatorassociated pneumonia (VAP) are among the most frequent nosocomial infections encountered in the ICU and are responsible for increases in length of stay, mortality and morbidity in critically ill patients. P. aeruginosa, along with the Enterobacteriacae, E. coli and K. pneumonia represent a substantial proportion of HAP and VAP infections. The use of empirical broad spectrum antibiotics for the treatment of pneumonia has led to an increase in antimicrobial resistance along with the development of multidrug resistant Gram-negative organisms.

Appropriate antibiotic exposure at the site of infection is an important component for therapy to be clinically effective. Epithelial lining fluid (ELF) is considered to be the site of infection for pneumonia and achieving sufficiently high drug concentrations in ELF are critical for treating pneumonia. The rationale for inhaling antibiotics is to maximize drug delivery to the target site of infection (ELF in the case of pneumonia) and limit the potential for systemic side effects. The uses of inhaled antimicrobials have a long history in the treatment of lower respiratory tract infections specifically in patients with cystic fibrosis [11]. Due to the increasing prevalence of resistant Gram-negative organisms in VAP causing difficult to treat pneumonia along with the limited lung concentrations that can be achieved with standard parentally given antibiotics there has been an increased interest in the use of inhaled antimicrobials such as inhaled amikacin for respiratory tract infections such as in HAP and VAP [12]. In a recently published study, So et al. [6] investigated the in vitro pharmacodynamics of human simulated ELF concentrations of inhaled amikacin against variety of phenotypically diverse susceptible and non-susceptible K. pneumoniae and $P$. aeruginosa isolates and showed rapid bactericidal activity against isolates with an amikacin MICs of $\leq 256 \mathrm{mg} / \mathrm{L}$. 
Table 2 MIC profile of AMK and comparators for isolates of E. coli and K. pneumoniae

\begin{tabular}{|c|c|c|c|c|c|c|}
\hline Isolates & Antimicrobial agent & Range & Modal & $\mathrm{MIC}_{50}$ & $\mathrm{MIC}_{90}$ & $\% \mathrm{~S}$ \\
\hline All isolates & AMK & $\leq 0.5->128$ & 2 & 4 & 16 & 96 \\
\hline \multirow[t]{10}{*}{$n=1646$} & ATM & $\leq 0.06->64$ & 0.06 & 0.125 & 32 & 85 \\
\hline & FEP & $\leq 0.06->64$ & 0.06 & 0.06 & 32 & 87 \\
\hline & CAZ & $\leq 0.06->64$ & 0.25 & 0.25 & 64 & 85 \\
\hline & $\mathrm{C} / \mathrm{T}$ & $\leq 0.06->64$ & 0.25 & 0.25 & 1 & 94 \\
\hline & CRO & $\leq 0.06->64$ & 0.06 & 0.06 & 128 & 84 \\
\hline & $\mathrm{CIP}$ & $\leq 0.015->16$ & 0.015 & 0.06 & 32 & 74 \\
\hline & IPM & $\leq 0.06->64$ & 0.25 & 0.25 & 1 & 96 \\
\hline & MEM & $\leq 0.06->64$ & 0.06 & 0.06 & 0.06 & 96 \\
\hline & TZP & $\leq 0.25->256$ & 2 & 4 & 32 & 88 \\
\hline & TOB & $\leq 0.06->64$ & 0.5 & 1 & 16 & 84 \\
\hline ESBL+isolates & AMK & $0.5->128$ & 16 & 8 & 32 & 87 \\
\hline \multirow[t]{10}{*}{$n=173$} & ATM & $\leq 0.06->64$ & 64 & 64 & 128 & 9 \\
\hline & FEP & $\leq 0.06->64$ & 128 & 128 & 128 & 16 \\
\hline & CAZ & $0.125->64$ & 128 & 64 & 128 & 14 \\
\hline & $\mathrm{C} / \mathrm{T}$ & $\leq 0.06->64$ & 0.5 & 1 & 8 & 79 \\
\hline & $\mathrm{CRO}$ & $\leq 0.06->64$ & 128 & 128 & 128 & 5 \\
\hline & CIP & $\leq 0.015->16$ & 32 & 32 & 32 & 16 \\
\hline & IPM & $0.125->64$ & 0.25 & 0.25 & 1 & 94 \\
\hline & MEM & $\leq 0.06->64$ & 0.06 & 0.06 & 0.125 & 95 \\
\hline & TZP & $0.25->256$ & 4 & 8 & 256 & 62 \\
\hline & TOB & $0.25->64$ & 32 & 16 & 64 & 39 \\
\hline FEP-R isolates & AMK & $0.5->64$ & 8 & 8 & 64 & 79 \\
\hline \multirow[t]{10}{*}{$n=216$} & ATM & $0.125->64$ & 128 & 64 & 128 & 6 \\
\hline & FEP & $4->64$ & 128 & 128 & 128 & 0 \\
\hline & CAZ & $0.5->64$ & 128 & 128 & 128 & 9 \\
\hline & $\mathrm{C} / \mathrm{T}$ & $0.125->64$ & 0.5 & 1 & 128 & 59 \\
\hline & $\mathrm{CRO}$ & $\leq 0.06->64$ & 128 & 128 & 128 & 5 \\
\hline & $\mathrm{CIP}$ & $\leq 0.015->16$ & 32 & 32 & 32 & 13 \\
\hline & IPM & $0.06->64$ & 0.25 & 0.25 & 32 & 75 \\
\hline & MEM & $\leq 0.06->64$ & 0.06 & 0.06 & 32 & 75 \\
\hline & TZP & $0.25->256$ & 512 & 32 & 512 & 46 \\
\hline & TOB & $0.25->64$ & 32 & 16 & 64 & 32 \\
\hline TZP-R isolates & AMK & $0.5->64$ & 16 & 8 & 64 & 78 \\
\hline \multirow[t]{10}{*}{$n=191$} & ATM & $\leq 0.06->64$ & 128 & 32 & 128 & 36 \\
\hline & FEP & $\leq 0.06->64$ & 128 & 16 & 128 & 39 \\
\hline & CAZ & $0.25->64$ & 128 & 64 & 128 & 33 \\
\hline & $\mathrm{C} / \mathrm{T}$ & $\leq 0.06->64$ & 128 & 1 & 128 & 56 \\
\hline & $\mathrm{CRO}$ & $\leq 0.06->64$ & 128 & 128 & 128 & 35 \\
\hline & $\mathrm{CIP}$ & $\leq 0.015->16$ & 32 & 32 & 32 & 34 \\
\hline & IPM & $0.125->64$ & 0.25 & 0.5 & 32 & 70 \\
\hline & MEM & $\leq 0.06->64$ & 0.06 & 0.06 & 32 & 71 \\
\hline & TZP & $32->256$ & 512 & 256 & 512 & 0 \\
\hline & TOB & $0.025->64$ & 32 & 16 & 64 & 39 \\
\hline
\end{tabular}

AMK amikacin; ATM aztreonam; FEP cefepime; CAZ ceftazidime; C/T ceftolozane/tazobactam; CRO ceftriaxone, CIP ciprofloxacin; IPM imipenem; MEM meropenem; TZP piperacillin/tazobactam; TOB tobramycin

The high overall susceptibility profile of amikacin against these Gram-negative organisms of interest in our current study appears to represent the sustained in vitro potency of the compound when compared to two previously reported surveillance programs $[13,14]$. The INFORM study collected 7062 clinical $P$. aeruginosa isolates from 


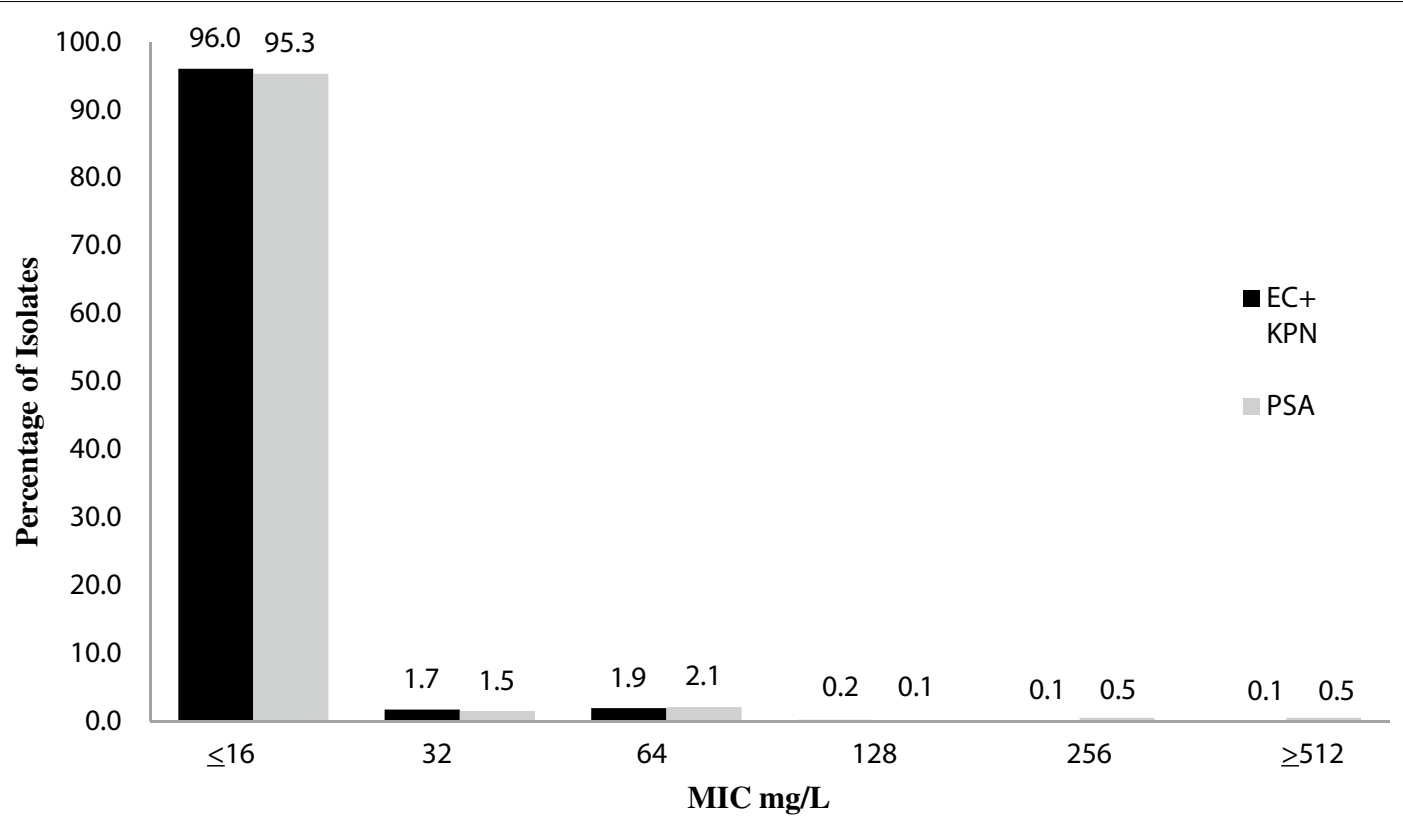

Fig. 1 Distribution of Amikacin (AMK) against E. coli (EC) and K. pneumoniae (KPN) versus P. aeruginosa (PSA)

Europe, Asia/South Pacific, Latin America and Middle East/Africa [13]. Similar to our data the INFORM global surveillance program found amikacin to have a high susceptibility $(89.4 \%)$ against the $P$. aeruginosa isolates tested. Moreover, the authors also reported similar $( \pm 6 \%)$ susceptibility profiles for ceftazidime, cefepime, piperacillintazobactam and meropenem to that in our current study.

The SMART surveillance program collected intraabdominal isolates of $E$. coli, K. pneumoniae, K. oxytoca and P. mirabilis [14]. E. coli $(\mathrm{n}=434)$ maintained high susceptibilities to amikacin of $>99 \%$, while K. pneumoniae $(\mathrm{n}=231)$ had $93.5 \%$. Both organisms had $\geq 92 \%$ susceptibility to imipenem and ertapenem. In the SMART program amikacin retained the highest susceptibility to MDR Enterobacteriaceae of all the agents tested.

\section{Conclusions}

In this study we defined the phenotypic profile of amikacin against 2460 blood and respiratory nosocomial isolates implicated in ICU based pulmonary infections collected from 50 US hospitals. AMK demonstrated a high level of activity to combat infections caused by the Enterobacteriacae, E. coli and $K$. pneumoniae including those strains producing ESBLs. When considering E. coli and K. pneumoniae our study found amikacin to have an $\mathrm{MIC}_{50}$ and $\mathrm{MIC}_{90}$ of 4 and $16 \mathrm{mg} / \mathrm{L}$, respectively. Moreover, $96 \%$ of organisms had a MIC of $\leq 16 \mathrm{mg} / \mathrm{L}$ and nearly all (99\%) organisms had MICs $\leq 64 \mathrm{mg} / \mathrm{L}$ despite concomitant $\beta$-lactam or fluoroquinolone resistance. In a study conducted by Sader et al. [15] analyzing the in vitro activity of amikacin against isolates gathered from patients hospitalized with pneumonia including VAP, across 62 US hospitals, the $\mathrm{MIC}_{50}$ and $\mathrm{MIC}_{90}$ was reported to be 1 and $32 \mathrm{mg} / \mathrm{L}$, respectively, against K. pneumoniae. In a study investigating $E$. coli isolates across 66 Canadian medical centers the reported amikacin $\mathrm{MIC}_{50}$ and $\mathrm{MIC}_{90}$ were $\leq 2$ and $4 \mathrm{mg} / \mathrm{L}$, respectively [16].

For $P$. aeruginosa, our study found amikacin to have an $\mathrm{MIC}_{50}$ and $\mathrm{MIC}_{90}$ of 8 and $16 \mathrm{mg} / \mathrm{L}$, respectively. Against these isolates $95 \%$ of organisms had amikacin MICs of $\leq 16 \mathrm{mg} / \mathrm{L}$, moreover nearly all (97\%) organisms have MICs $\leq 32 \mathrm{mg} / \mathrm{L}$. Similarly when compared to the Sader et al. [15] study, P. aeruginosa isolates from 62 US hospitals, amikacin was reported to have an $\mathrm{MIC}_{50}$ and $\mathrm{MIC}_{90}$ of 4 and $8 \mathrm{mg} / \mathrm{L}$, respectively. Another study analyzing similar data from 127 isolates from Canadian hospitals, reported $\mathrm{MIC}_{50}$ and $\mathrm{MIC}_{90}$ of 4 and $16 \mathrm{mg} / \mathrm{L}$, respectively [17].

With respect to resistant Gram-negative bacteria, our study demonstrated that amikacin maintained potent activity against $P$. aeruginosa MDR organisms. In regards to ESBLs this study showed that AMK was a potent antimicrobial with a high level of activity against these MDR isolates as $87 \%$ had an MIC of $\leq 16 \mathrm{mg} / \mathrm{L}$. Against MDR P. aeruginosa isolates, amikacin was the most potent antibiotic tested as $87 \%$ of resistant isolates were considered susceptible (i.e., $\mathrm{MIC}$ of $\leq 16 \mathrm{mg} / \mathrm{L}$ ).

The current surveillance study which incorporated a large number of US hospitals demonstrated the high potency of amikacin against contemporary isolates of E. coli, K. pneumoniae, P. aeruginosa originating from a 
Table 3 MIC profile of AMK and comparators for isolates of $P$. aeruginosa

\begin{tabular}{|c|c|c|c|c|c|c|}
\hline Isolates & Antimicrobial agent & Range & Modal & $\mathrm{MIC}_{50}$ & $\mathrm{MIC}_{90}$ & $\% \mathrm{~S}$ \\
\hline All isolates & AMK & $\leq 0.5->64$ & 4 & 8 & 16 & 95 \\
\hline \multirow[t]{9}{*}{$\mathrm{n}=814$} & ATM & $\leq 0.06->64$ & 4 & 8 & 32 & 67 \\
\hline & FEP & $\leq 0.06->64$ & 2 & 4 & 32 & 73 \\
\hline & CAZ & $0.125->64$ & 2 & 4 & 64 & 74 \\
\hline & $C / T$ & $\leq 0.06->64$ & 0.5 & 0.5 & 4 & 95 \\
\hline & $\mathrm{CIP}$ & $\leq 0.015->16$ & 0.125 & 0.25 & 16 & 68 \\
\hline & IPM & $\leq 0.06->64$ & 1 & 2 & 16 & 62 \\
\hline & MEM & $\leq 0.06->64$ & 0.5 & 1 & 16 & 71 \\
\hline & TZP & $\leq 0.25->256$ & 8 & 8 & 256 & 67 \\
\hline & TOB & $\leq 0.06->64$ & 0.5 & 1 & 8 & 90 \\
\hline MDR+isolates & AMK & $0.5->64$ & 8 & 8 & 32 & 87 \\
\hline \multirow[t]{9}{*}{$n=116$} & ATM & $0.25->64$ & 128 & 32 & 128 & 19 \\
\hline & FEP & $2->64$ & 32 & 32 & 128 & 13 \\
\hline & CAZ & $2->64$ & 128 & 64 & 128 & 16 \\
\hline & $C / T$ & $0.5->64$ & 1 & 2 & 32 & 78 \\
\hline & CIP & $2->64$ & 32 & 16 & 32 & 11 \\
\hline & IPM & $0.5->64$ & 32 & 16 & 32 & 11 \\
\hline & MEM & $0.25->64$ & 16 & 16 & 64 & 17 \\
\hline & TZP & $4->256$ & 256 & 256 & 512 & 6 \\
\hline & TOB & $0.125->64$ & 1 & 4 & 128 & 52 \\
\hline MEM-R isolates & AMK & $0.5->64$ & 8 & 8 & 16 & 91 \\
\hline \multirow[t]{9}{*}{$n=236$} & ATM & $0.25->64$ & 32 & 16 & 64 & 35 \\
\hline & FEP & $1->64$ & 16 & 16 & 64 & 42 \\
\hline & CAZ & $0.5->64$ & 128 & 16 & 128 & 47 \\
\hline & $C / T$ & $0.25->64$ & 1 & 1 & 8 & 89 \\
\hline & $\mathrm{CIP}$ & $0.06->16$ & 32 & 4 & 32 & 35 \\
\hline & IPM & $0.25->64$ & 16 & 16 & 32 & 11 \\
\hline & MEM & $4->64$ & 16 & 16 & 32 & 0 \\
\hline & TZP & $4->256$ & 32 & 32 & 512 & 36 \\
\hline & TOB & $\leq 0.06->64$ & 1 & 1 & 64 & 74 \\
\hline FEP-R isolates & AMK & $0.5->64$ & 8 & 8 & 32 & 89 \\
\hline \multirow[t]{9}{*}{$n=222$} & ATM & $0.25->64$ & 32 & 32 & 128 & 23 \\
\hline & FEP & $16->64$ & 16 & 32 & 64 & 0 \\
\hline & CAZ & $2->64$ & 128 & 32 & 128 & 26 \\
\hline & $C / T$ & $0.25->64$ & 1 & 2 & 8 & 85 \\
\hline & $\mathrm{CIP}$ & $0.06->16$ & 32 & 4 & 32 & 39 \\
\hline & IPM & $0.5-64$ & 16 & 8 & 32 & 37 \\
\hline & MEM & $\leq 0.06->64$ & 16 & 8 & 32 & 38 \\
\hline & TZP & $1->256$ & 256 & 128 & 512 & 20 \\
\hline & TOB & $0.25->64$ & 1 & 1 & 64 & 76 \\
\hline TZP-R isolates & AMK & $0.5->64$ & 8 & 8 & 16 & 91 \\
\hline \multirow[t]{8}{*}{$n=267$} & ATM & $0.25->64$ & 32 & 32 & 128 & 27 \\
\hline & FEP & $1->64$ & 16 & 16 & 64 & 34 \\
\hline & CAZ & $0.5->64$ & 128 & 32 & 128 & 31 \\
\hline & $\mathrm{C} / \mathrm{T}$ & $0.25->64$ & 1 & 2 & 8 & 88 \\
\hline & $\mathrm{CIP}$ & $\leq 0.015->16$ & 32 & 2 & 32 & 46 \\
\hline & IPM & $0.125->64$ & 32 & 8 & 32 & 39 \\
\hline & MEM & $0.125->64$ & 16 & 4 & 32 & 44 \\
\hline & TZP & $32->256$ & 32 & 128 & 512 & 0 \\
\hline
\end{tabular}


Table 3 continued

\begin{tabular}{lccccc}
\hline Isolates & Antimicrobial agent & Range & Modal & MIC $_{\mathbf{5 0}}$ & MIC $_{\mathbf{9 0}}$ \\
\hline TOB & $0.25->64$ & 1 & 1 \\
\hline
\end{tabular}

AMK amikacin; ATM aztreonam; FEP cefepime; CAZ ceftazidime; C/T ceftolozane/tazobactam; CIP ciprofloxacin; IPM imipenem; $M E M$ meropenem; TZP piperacillin/ tazobactam; TOB obramycin

blood or respiratory source. Moreover, despite resistance to representative agents from the $\beta$-lactam and fluoroquinolone classes, AMK maintained high susceptibility for all organisms. As a result of low MIC's and high achievable lung concentrations of approximately $5000 \mathrm{mg} / \mathrm{L}$, Amikacin Inhale has a potential adjunctive role in the management of bronchopulmonary infections caused by $P$. aeruginosa, E. coli and $K$. pneumoniae in the intubated patient. While Amikacin Inhale appears to be a viable adjunctive therapy, patients should also receive appropriate systemic antimicrobial therapy based on local susceptibilities or when available the susceptibility profile of the patient specific isolate(s).

\begin{abstract}
Abbreviations
HAP: hospital-acquired pneumonia;VAP: ventilator-associated pneumonia; ICU: intensive care unit; P. aeruginosa: Pseudomonas aeruginosa, E. coli: Escherichia coil; K. pneumoniae: Klebsiella pneumoniae; CT: Connecticut; US: United States; AMK: amikacin; ELF: epithelial lining fluid; S. maltophilia: Stenotrophomonas maltophilia; CLSI: Clinical Laboratory Standards Institute; MICs: minimum inhibitory concentration; ATM: aztreonam; FEP: cefepime; CAZ: ceftazidime; C/T: ceftolozane/tazobactam; CRO: ceftriaxone; CIP: ciprofloxacin; IPM: imipenem; MEM: meropenem; TZP: piperacillin/tazobactam; TOB: tobramycin; MO: Missouri; FDA: food and drug administration; MDR: multidrug resistant; ESBL: extended spectrum $\beta$-lactamases; K. oxytoca: Klebsiella oxytoca; P. mirabilis: Proteus mirabilis.
\end{abstract}

\section{Authors' contributions}

CS and JV were involved in all aspects of this study including data collection, analysis and manuscript preparation. DPN was the principal investigator on this study. All authors read and approved the final manuscript.

\section{Author details}

${ }^{1}$ Center for Anti-Infective Research and Development, Hartford Hospital, 80 Seymour Street, Hartford, CT 06102, USA. ${ }^{2}$ Division of Infectious Diseases, Hartford Hospital, Hartford, CT, USA.

\section{Acknowledgements}

We would like to thank Jennifer Tabor-Renniel, Lucinda Lamb, Sara Robinson, Debora Santini, Kimelyn Greenwood and Elizabeth Cyr for their collective efforts with MIC determination.

\section{Competing interests}

David Nicolau has received grants and is on the speakers' bureau for Bayer Pharma. Christina Sutherland and Jamie Verastegui have no competing interests.

\section{Availability of data and materials}

Data will be shared upon request to the corresponding author david.nicolau@ hhchealth.org.

\section{Funding}

This study was supported by Bayer Pharma AG, Berlin, Germany.

Received: 18 April 2016 Accepted: 8 June 2016

Published online: 17 June 2016

\section{References}

1. Pfaller MA, Mendes RE, Sader HS, Jones RN. Telavancin activity against Gram-positive bacteria isolated from respiratory tract specimens of patients with nosocomial pneumonia. J Antimicrob Chemother. 2010;65(11):2396-404.

2. American Thoracic Society; Infectious Diseases Society of America. Guidelines for the management of adults with hospital-acquired, ventilatorassociated and healthcare-associated pneumonia. Am J Respir Crit Care Med. 2005;171(4):388-416.

3. Barbier F, Andremont A, Wolff M, Bouadma L. Hospital-acquired pneumonia and ventilator-associated pneumonia: recent advances in epidemiology and management. Curr Opin Pulm Med. 2013;19(3):216-28.

4. Charles MP, Easow JM, Joseph NM, Ravishankar M, Kumar S, Sivaraman U. Aetiological agents of ventilator-associated pneumonia and its resistance pattern — a threat for treatment. Australasian Med J. 2013;6(9):430-4.

5. Bayer HealthCare AG. Amikacin inhalation solution and the pulmonary drug delivery system [PDDS Clinical] Gram-negative pneumonia. 2012;08 Nov.

6. So W, Crandon JL, Hamada Y, Nicolau DP. Antibacterial activity of achievable epithelial lining fluid exposures of Amikacin Inhale with or without meropenem. J Antimicrob Chemother. 2016;2016(71):428-37.

7. Clinical and Laboratory Standards Institute. 2014. Performance standards for antimicrobial susceptibility testing; twenty-fourth informational supplement. CLSI document M100-S24 U. Clinical and Laboratory Standards Institute, Wayne, PA.

8. ZERBAXA (ceftolozane/tazobactam) for injection, for intravenous use. 2014. Initial U.S. Approval: 2014. Cubist Pharmaceuticals.

9. Bulik CC, Christensen H, Nicolau DP. In vitro potency of CXA-101, a novel cephalosporin, against Pseudomonas aeruginosa displaying various resistance phenotypes, including multidrug resistance. Antimicrob Agents Chemother. 2010;54(1):557-9.

10. Dortet L, Poirel L, Nordmann P. Rapid identification of carbapenemase types in Enterobacteriaceae and Pseudomonas spp. by using a biochemical test. Antimicrob Agents Chemother. 2012;56:6437-40.

11. Quon BS, Goss CH, Ramsey BW. Inhaled antibiotics for lower airway infections. Ann Am Thorac Soc. 2014;11:425-34

12. Nicolau DP, Dimopoulos G, Welte T, Luyt CE. Can we improve clinical outcomes in patients with pneumonia treated with antibiotics in the intensive care unit? Expert Rev Respir Med. 2016:3:1-12. doi:10.1080/1747 6348.2016.1190277.

13. Nichols WW, de Jonge BL, Kazmierczak KM, Karlowsky JA, Sahm DF. In vitro susceptibility of global surveillance isolates of Pseudomonas aeruginosa to ceftazidime-avibactam: INFORM 2012-2014. Antimicrob Agents Chemother. 2016. doi:10.1128/AAC.00220-16.

14. Zalacain M, Biedenbach DJ, Badal RE, Young K, Motyl M, Sahm DF. Pathogen prevalenceand antimicrobial susceptibility among enterobacteriaceae causing hospital-associated intra-abdominal infections in adults in the United States (2012-2013). Clin Ther. 2016. doi:10.1016/j. clinthera.2016.04.035.

15. Sader HS, Rhomberg PR, Farrell DJ, Jones RN. Arbekacin activity against contemporary clinical bacteria isolated from patients hospitalized with pneumonia. Antimicrob Agents Chemother. 2015;59(6):3263-70.

16. Zhanel, George G, et al. Antimicrobial susceptibility of 15,644 pathogens from Canadian hospitals: results of the CANWARD 2007-2009 study. Diagn Microbiol Infect Dis. 2011;69:291-306.

17. Zelenitsky SA, Rubinstein E, Ariano R, et al. Integrating pharmacokinetics, pharmacodynamics and MIC distribution to assess changing antimicrobial activity against clinical isolates of Pseudomonas aeruginosa causing infections in Canadian hospitals (CANWARD). J Antimicrob Chemother. 2013;68(Suppl 1):67-72. 